\title{
O.S.P.
}

\section{Territoires scolaires, incivilités dans les lycées et normativités adolescentes}

School territories, anti-social behavior in secondary schools and adolescent normativities

Marie-Line Félonneau et Lyda Lannegrand-Willems

\section{(2) OpenEdition}

\section{Journals}

Édition électronique

URL : http://journals.openedition.org/osp/2158

DOI : 10.4000/osp.2158

ISSN : 2104-3795

Éditeur

Institut national d'étude du travail et d'orientation professionnelle (INETOP)

Édition imprimée

Date de publication : 15 juin 2004

Pagination : 271-287

ISSN : 0249-6739

Référence électronique

Marie-Line Félonneau et Lyda Lannegrand-Willems, «Territoires scolaires, incivilités dans les lycées et normativités adolescentes ", L'orientation scolaire et professionnelle [En ligne], 33/2 | 2004, mis en ligne le 15 décembre 2009, consulté le 19 avril 2019. URL : http://journals.openedition.org/osp/2158 ; DOI : $10.4000 /$ osp. 2158

Ce document a été généré automatiquement le 19 avril 2019.

(c) Tous droits réservés 


\section{Territoires scolaires, incivilités dans les lycées et normativités adolescentes ${ }^{1}$}

School territories, anti-social behavior in secondary schools and adolescent normativities

Marie-Line Félonneau et Lyda Lannegrand-Willems

\section{Introduction}

1 À partir d'un prisme théorique associant la psychologie sociale environnementale et la psychologie du développement, nous proposons une lecture psychosociale de l'inscription de l'adolescent dans un des espaces majeurs de sa vie quotidienne : le lycée.

2 D'emblée, nous posons que les représentations spatiales et les pratiques d'usage de l'espace scolaire sont constitutives des processus identitaires à l'adolescence. Des travaux précédents sur cette thématique (Felonneau, 1997; Felonneau \& Busquets, 2001) ont révélé un profond clivage entre les représentations et les pratiques de l'espace selon que celui-ci est contraint comme l'espace scolaire ou non contraint et ludique comme la ville. En effet, dans chacun de ces contextes, les jeunes projettent des rôles différents joués en compagnie des pairs et face aux adultes et mettent en scène leur vie quotidienne en accédant à l'urbanité.

3 Notre approche de la spatialisation de l'identité postule une certaine spécificité psychologique et sociale de l'adolescence. En effet, à défaut de trouver les repères identificatoires dans la société et les institutions, à défaut de devoir se soumettre à des rituels sociaux qui débouchaient traditionnellement sur la reconnaissance sociale, les adolescents inventent de nouveaux codes, langagiers, vestimentaires, culturels, sociaux.

4 L'émission de comportements déviants quant à la norme ${ }^{2}$ (communément appelés incivilités) sera ici considérée comme un révélateur de la nature des relations intergroupes (adolescents/enseignants; adolescents/parents). Analyser l'usage de 
l'espace scolaire par les lycéens permet ainsi probablement de dévoiler l'existence de rituels d'appartenance comme cracher parterre par exemple ou taguer les murs !

\section{Territorialités scolaires, spatialisation de l'identité et nouvelles normativités lycéennes}

\section{Le lycée est-il un territoire?}

On doit aux premiers psychologues de l'environnement les notions de territoire et de territorialité. On leur doit aussi quelques éclaircissements théoriques sur la notion jumelle de territoire, l'appropriation.

6 Selon Altman (1975), la territorialité implique tout simplement l'utilisation exclusive des espaces et des objets par des personnes et des groupes. Elle se déploie au travers d'« un ensemble de conduites spatiales exprimant plus ou moins explicitement un contrôle symbolique sur un espace donné et par là même un certain type de rapport à l'environnement et aux groupes. C'est cet ensemble de pratiques, qui, lorsqu'elles sont identifiées, révèlent les contours du territoire d'un sujet ou d'un groupe » (Félonneau, 1997, p. 46).

7 La notion de territoire suppose une zone d'emprise à laquelle on peut s'identifier ; c'est un des supports des processus de spatialisation identitaire. Les psychologues y voient même une extension du moi. Fischer (1992) parle, quant à lui, de champ topologique et insiste sur le découpage d'un espace physique en zones subjectives délimitées par la qualité des relations établies avec autrui. Le concept de territoire désigne aussi cet usage que nous faisons des lieux selon les significations psychologiques et culturelles que leur confèrent les cadres sociaux.

8 Pour les adolescents, le lycée est un territoire secondaire ${ }^{3}$, un lieu d'échanges sociaux régis par des codes, rituels et certaines règles plus ou moins formelles. Le lycée est, en quelque sorte, la figure inversée de la ville qui est un territoire public, ouvert, accessible. Il reste le lieu obligé de la soumission et de la contrainte. Pour autant, comme le montrent des travaux portant sur les représentations socio-spatiales des jeunes (Félonneau, 1997) ou sur les modalités de l'expérience scolaire adolescente (Dubet, 1991), le lycée constitue, à l'instar de tous les espaces de travail, un espace irréductible qui ne peut être entièrement soumis dès lors qu'il fait l'objet d'une appropriation, si minimale soit-elle.

9 À partir de la notion d'appropriation, on peut analyser des schèmes comportementaux exprimant concrètement des formes d'agir, de sentir qui témoignent à la fois d'une emprise sur les lieux et de la production de normes d'usages de l'espace spécifiques. Or l'appropriation introduit toujours une perturbation relative, voire une certaine forme de subversion dans les rouages prétendument rationnels des organisations. L'adolescent, comme l'adulte, est confronté à " la gestion de soi-même dans des lieux qui sont apparemment déjà gérés entièrement pour lui» (Fischer, 1989, p. 141). Ainsi, les adolescents au lycée essaieront de marquer l'espace de leur empreinte pour manifester en quelque sorte une forme de résistance au monde des adultes. Certes, l'existence d'un fossé entre la génération des parents et celle des adolescents est mise en cause dans de nombreux travaux de psychologie de l'adolescence. La mise en évidence de cultures adolescentes plurielles va à l'encontre de l'image populaire d'une culture adolescente homogène opposée aux valeurs des adultes (Brown, 1990 ; Steinberg \& Morris, 2001), et l'on observe fréquemment un chevauchement entre les valeurs du groupe de pairs et les valeurs des 
parents (Conger, 1991). Néanmoins, une grande majorité des jeunes adoptent des comportements dérogatoires au cours de l'adolescence (Coslin, 1999; Farrington, 1995) qui peuvent être envisagés comme des questionnements des conduites et des pratiques sociales: l'adolescent questionne ainsi la conformité des comportements qui lui sont proposés ou imposés par la société et dont il n'a pas participé à l'élaboration (Coslin, 2002).

Les pairs prennent une place prépondérante dans l'univers social des adolescents notamment au milieu de la période adolescente - entre 14 et 17 ans - (Buhrmester, 1996; Furman \& Whener, 1997), période où l'influence entre pairs est la plus forte (Brown, 1990). Or, le lycée est un des lieux où se donne à voir quotidiennement le groupe d'appartenance générationnel. Il est un des lieux majeurs de l'expérience sociale (Dubet, 1991) des adolescents, lieu de rencontres et d'échanges qui incarne en quelque sorte une communauté de destin (Maffesoli, 2000) au moins de façon transitoire. Même si l'idée de communauté ne semble plus guère convenir au lycée d'aujourd'hui, tant il paraît parfois anomique, il n'en reste pas moins qu'il est le lieu où s'expérimentent, parfois dans la douleur (Dubet, 1991 ; Dumora, 1998), les processus d'élimination et de sélection à l'œuvre dans la société globale. C'est dans l'espace scolaire que se joue l'image de soi, et se construit l'identité psychosociale de l'élève (Lannegrand-Willems, 2000).

\section{La spatialisation identitaire à l'adolescence}

11 L'environnement familier, en l'occurrence le lycée, est posé comme un espace d'inscription du Soi caractérisé par sa fonction majeure d'étayage identitaire.

S'approprier les lieux de la vie quotidienne, surtout ceux que l'on est obligé de partager avec autrui, se sentir chez soi, c'est partager les mêmes codes d'usage de l'espace commun. Car à défaut d'incarner les valeurs légitimes de la culture (Bourdieu, 1979), le lycée d'aujourd'hui continue d'exprimer une identité adolescente qui s'y construit jour après jour dans les interactions quotidiennes tant avec les pairs qu'avec les adultes.

13 Pour que s'opèrent les remaniements identitaires propres à cette période de la vie, les adolescents vont définir des usages spatiaux fonctionnant comme des marqueurs identitaires qui sont autant d'actes et de dispositifs ayant pour fonction de manifester et de poser une revendication sur une partie d'un territoire (Goffman, 1973).

14 Quels sont les nouveaux repères symboliques, les nouvelles normativités que choisira le sujet adolescent pour s'approprier son espace de vie et le disputer à l'ordre adulte en quelque sorte?

\section{Normativités adolescentes et incivilités}

15 Depuis les travaux fondateurs de Shérif (1965) sur la normalisation, les notions de norme et de normativité constituent les fondements théoriques de la psychologie sociale. La normativité est, comme la conformisation, la mise en place et le respect d'une norme groupale. Nous avons tenté de mettre au jour une norme adolescente concernant l'évaluation (en termes de gravité versus non gravité) de certains comportements qualifiés communément d'incivilités.

16 Le respect d'un certain nombre de codes sociaux et de rituels comportementaux constitue les fondements de la civilité qu'il convient de définir comme un mode de gestion 
harmonieuse des échanges sociaux quotidiens (Bernard, 1997). À l'inverse, leur nonobservance définit l'incivilité ou plus justement les incivilités, qui, par définition, sont plurielles. Nous avons cherché à savoir ce qui, en matière d'incivilités, était plus ou moins graves, aux yeux des adolescents. Pour éviter d'utiliser dans les questionnaires le terme de gravité trop connoté de notions morales voire moralistes et susceptibles de générer des biais de réactance chez les sujets, nous y avons substitué celui d'excusabilité. Nous avons suivi en cela l'exemple du groupe de sociologues européens qui travaillent depuis 1981 sur les transformations des systèmes de valeurs à l'échelle de l'Europe (Riffault, 1993; Scardigli, 1987 ; Stoetzel, 1983) ${ }^{4}$.

17 La thématique des incivilités, largement développée aux États-Unis depuis les années 70, contemporaine de la «tolérance zéro" reste d'une brûlante actualité en France notamment avec l'émergence d'une politique «musclée » contre l'insécurité. Au-delà du flou sémantique de la notion d'incivilité et de son utilisation partisane, il convient de se doter d'une définition simple et opératoire. Dans le cadre de cet article, les incivilités seront définies comme des actes d'irrespect ou d'agression envers autrui et l'environnement, n'entrant pas obligatoirement dans la catégorie juridique de la délinquance et du crime (Furstenberg, 1971; Lagrange, 1984, 1995 ; Roché, 1996, 1998). Ces comportements sont généralement peu graves mais deviennent intolérables au quotidien (Wievorka, 1999) parce qu'ils déclenchent un sentiment diffus de déclin de l'ordre social et moral et donc de peur (Debarbieux, 1996 ; Moser \& Lidvan 1991). Dans le cadre scolaire, ils ont surtout pour conséquences de nuire au cours normal des apprentissages mais aussi d'une façon plus diffuse - et non moins insupportable - de ruiner la qualité de l'ambiance générale.

18 En posant que les règles de civilité sont des conventions partagées concernant les actes usuels de la vie quotidienne, c'est bien dans le cadre d'une normativité donnée et relative qu'il faut resituer et définir les incivilités comme manquements aux allants de soi et aux routines définissant l'interaction normale dans l'espace scolaire.

19 Il s'agira de montrer que ces manquements aux normes peuvent s'analyser comme des « offenses territoriales» (Goffman, 1973) exprimant plus ou moins consciemment le désir des lycéens de s'affranchir des conventions adultes. Pour cela, on peut imaginer l'existence de véritables rituels comportementaux qui signent à la fois l'appartenance à l'endogroupe adolescent et la résistance à l'exogroupe adulte. Certes, aujourd'hui, compte tenu de la relativisation générale des interdits (Ehrenberg, 1998), on constate un infléchissement de l'autorité formelle, "on est loin de la petite guerre froide entre les surveillants généraux, les pions, les élèves, les anciens et les nouveaux qui faisait l'ordinaire du lycée» (Dubet, 1991, p. 289) ; cependant, les élèves, d'une façon générale, s'estiment contraints par le cadre scolaire et par ses exigences. L'extension des droits et des libertés qu'on leur accorde par ailleurs (notamment dans la famille) n'en rend que plus coercitive la discipline - pourtant allégée - de la sphère scolaire.

L'adolescence étant considérée comme une période à risques (Cloutier, 1996) au cours de laquelle les comportements dérogatoires sont plus fréquents que chez les jeunes adultes et les adultes (Coslin, 2002; Steinberg, 1999), l'adoption de certains comportements déviants - incivils - soit vis-à-vis d'autrui soit vis-à-vis de l'environnement physique pourrait être interprétée comme une certaine forme d'idiome adolescent ayant pour fonction implicite l'étayage identitaire et pour fonction explicite l'assouvissement des besoins d'affiliation et de singularisation propres à cette phase du développement. Cet idiome pourtant est à décrypter au préalable et le chercheur est bien souvent surpris de 
constater que ce qu'il considère comme une incivilité n'en est pas une pour les jeunes et inversement. C'est pour cette raison que nous avons travaillé sur un dictionnaire des incivilités produit par les jeunes eux-mêmes. Des travaux montrent que se conduire de façon incivile renvoie à la tentative de se dégager de l'emprise adulte pour parvenir à la reconnaissance de ses pairs (Leblanc \& Frechette, 1983 ; Selosse, 1997). Mais l'analyse du sens de ce langage ne peut se faire en-dehors de la question de la problématique identitaire à l'adolescence (Lipiansky, 1993).

Nous avons cherché à repérer l'existence d'une normativité adolescente spécifique, c'està-dire différente de celle des adultes, parents et enseignants. Pour ce faire, il s'agissait de se doter des outils méthodologiques susceptibles de mesurer la distance normative entre les différents types d'acteurs.

\section{Problématique}

22 À l'instar des travaux portant sur les usages que les jeunes font de l'espace (Augustin, 1994 ; Félonneau, 1994 ; Rouleau-Berger, 1991), nous posons que les traces imprimées dans l'espace par les adolescents, en l'occurrence les incivilités, peuvent être appréhendées comme des mécanismes de régulation du rapport aux adultes, susceptibles de soutenir la construction identitaire. Plus précisément, il s'agit de montrer que les incivilités expriment la nature des relations inter et intragroupes.

Bien qu'ils symbolisent plus ou moins directement un type particulier de rapport à l'exogroupe des adultes, les comportements spatiaux qualifiés d'incivils (gestuelle, bruits, agitation, attroupements...) sont susceptibles de varier selon le statut social de l'établissement fréquenté (favorisé versus défavorisé). Il s'agit ici de rendre compte de la fréquence perçue des incivilités dans chaque contexte. Perçoit-on les incivilités comme plus ou moins fréquentes selon que l'on est dans un lycée de quartier favorisé versus défavorisé ?

Enfin, on s'attend également à observer des normativités différentes selon le statut social de l'établissement fréquenté (favorisé versus défavorisé). En d'autres termes, il s'agit de rendre compte de l'existence de différences inter-établissements en fonction de l'évaluation de la gravité des incivilités. Juge-t-on les comportements incivils plus ou moins graves selon le contexte scolaire?

\section{Méthodologie et terrain d'investigation}

\section{Construction des échelles de fréquence et d'excusabilité des incivilités}

- Pour explorer la question de l'émergence éventuelle de nouvelles normativités, la première phase de la recherche a consisté à reconstituer le champ des incivilités. À la suite d'une préenquête menée à partir de la méthode d'associations libres, nous avons obtenu un premier répertoire d'incivilités émanant des propositions de sujets lycéens. Ce répertoire a fait l'objet d'une analyse de contenu thématique et a conduit à la construction d'une échelle de fréquence perçue des incivilités rencontrées dans le lycée. Cette échelle comprend 22 items dont 8 sont des incivilités physiques ou environnementales (respect versus dégradation d'éléments matériels), 10 des incivilités sociales (respect versus irrespect d'autrui) et enfin, 4 des incivilités scolaires. Chaque item est évalué sur une échelle en 5 points, ce qui nous 
permettait d'associer à chacun un signifié ( 1 = incivilité jamais rencontrée dans le lycée ; $2=$ parfois $; 3=$ souvent $; 4=$ très souvent $; 5=$ systématiquement rencontrée). Ici, la consigne est volontairement formulée sans précision de temps étant donné que l'objectif poursuivi n'est pas de mesurer objectivement les incivilités mais de repérer les représentations de leur occurrence.

- La normativité est opérationnalisée à l'aide d'une échelle d'excusabilité des incivilités au lycée. En d'autres termes, il s'agissait de mettre en évidence un lien entre un type d'environnement spatial donné et la plus ou moins grande tolérance à l'égard des conduites inciviles. Le contexte sociospatial de la conduite incivile influence-t-il la plus ou moins grande tolérance à son égard ? Concrètement, cette échelle est constituée des 22 items d'incivilités rencontrées dans le lycée. Seule la consigne est modifiée dans cette présentation : après s'être prononcé sur la fréquence perçue des incivilités, le sujet devait évaluer le degré d'excusabilité de chaque incivilité grâce à une échelle en 4 points $(1=$ tout à fait excusable ; 2 = plutôt excusable ; 3 = plutôt inexcusable ; 4 = tout à fait inexcusable).

- Pour comprendre ensuite en quoi les comportements incivils traduisent bien un mode spécifique de relation aux adultes, nous avons demandé aux sujets de répondre de nouveau à cette échelle d'excusabilité, en se mettant d'abord à la place de leurs enseignants ("À ton avis, que diraient tes professeurs de chacun de ces comportements dans le lycée?»), puis à la place de leurs parents (" ̀̀ ton avis, que diraient tes parents de chacun de ces comportements dans le lycée?»). Cette méthode permet ainsi de mesurer les écarts perçus par les lycéens entre leurs propres normes et celles des adultes, parents et enseignants.

Il s'agit d'une méthode désormais classique dans les travaux portant sur la présentation de soi et la clairvoyance normative et qui consiste à demander aux sujets de se décentrer par rapport à leur propre situation «de faire comme si... » ou encore «se mettre à la place de... ». Bien que notre réflexion ne s'inscrive pas dans ce cadre théorique, il s'agit bien cependant d'activer une certaine forme de "clairvoyance normative " (Py \& Somat, 1991) de la part des élèves à qui l'on demande de répondre à la place des adultes, donc en principe d'identifier aussi précisément que possible la norme dominante. En d'autres termes, la transgression ne peut être interprétée que dans la mesure où la norme dominante est connue et reconnue comme telle. En effet, dans un contexte trop anomique où les repères seraient trop ambigus, la transgression ne pourrait plus être appréhendée comme la mise en place d'une «normativité de résistance». Or, cette méthode permet l'accès direct à cette information.

\section{Présentation de l'échantillon et procédure}

Choisir le niveau du lycée plutôt que celui du collège peut se comprendre par la prise en compte du niveau de développement des sujets. Juger les incivilités et se positionner quant aux normes sociales supposent un certain niveau de développement moral; or selon la conception de Kohlberg (1969) le stade conventionnel du jugement moral s'initie vers l'âge de 12 ans et conduit progressivement (approximativement à partir de 17 ans) à l'intériorisation du jugement moral, à la prise en compte de l'ordre social et de la loi. Le palier de la classe de seconde de lycée - qui correspond à l'âge de 16 ans - semble donc pertinent dans le cadre de cette étude.

Le choix des lycées s'est porté sur les deux établissements les plus favorisés de la ville et sur les deux lycées les moins socialement favorisés (d'après la répartition des catégories socioprofessionnelles des parents éditée par le service statistique rectoral), ce qui 
correspond à la répartition sociale suivante : $52,2 \%$ des élèves de lycées favorisés sont issus des classes sociales supérieures (à savoir cadres supérieurs et professions libérales), contre 13,2 \% des élèves des lycées défavorisés; 14,8 \% des élèves de lycées favorisés sont issus des milieux employés et ouvrier, contre $42,5 \%$ des élèves de lycées défavorisés. Notre population cible est ainsi constituée de 842 lycéens bordelais fréquentant quatre établissements scolaires sociologiquement contrastés : 2 lycées socialement favorisés, 2 lycées défavorisés. Volontairement, nous avons choisi des aires écologiques socialement contrastées de façon à mettre au jour les déterminations sociales du rapport à l'espace scolaire.

Cet échantillon se compose de 282 garçons et de 560 filles, et sa moyenne d'âge est de 16,7 ans (écart-type $=1,48$ ). Bien que la répartition des garçons et des filles soit disproportionnée dans l'ensemble de l'échantillon, elle reste stable d'un type d'établissement à l'autre : les établissements socialement favorisés comprennent ici $64 \%$ de filles et $36 \%$ de garçons, les établissements socialement défavorisés regroupent quant à eux $70 \%$ de filles et $30 \%$ de garçons. Les élèves des lycées défavorisés ont en moyenne 17 ans (avec un écart-type de plus ou moins 1,68 ) et sont sensiblement plus âgés que les élèves de lycées socialement favorisés (moyenne $=16,2$; écart-type $=1,06$ ). Cette différence d'âge peut s'expliquer par le fait que les élèves de milieux sociaux favorisés ont moins redoublé dans leur scolarité.

Au niveau de la procédure de la recherche, la passation des questionnaires a été réalisée de façon collective dans les salles de classe des établissements. L'ordre de présentation des échelles était le suivant: d'abord, les sujets devaient estimer la fréquence perçue des incivilités, puis ils devaient répondre aux échelles d'excusabilité des incivilités pour soi (consigne 1), pour les professeurs (consigne 2) et pour les parents (consigne 3).

\section{Validité du questionnaire d'incivilités}

Une analyse en composantes principales réalisée sur l'échelle d'excusabilité des incivilités a permis de mettre en évidence l'existence d'un facteur général d'excusabilité des incivilités (saturations variant de .39 à .70 ; valeur propre $=5,661 ; 31,45 \%$ de variance expliquée). Une rotation Varimax des axes a permis ensuite de dégager 3 dimensions (tableau 1). Une première dimension regroupe 7 items d'incivilités environnementales (valeur propre $=3,256 ; 18,09 \%$ de variance expliquée; alpha $=.80$ ), comme graver les tables (item 12) ou encore jeter des papiers par terre (item 22). La deuxième dimension est composée de 6 items relatifs à des incivilités en direction de l'adulte, et notamment ici, en rapport aux professeurs et à l'institution scolaire, comme l'insolence envers les enseignants (item 3) ou les perturbations de cours (item 13) (valeur propre $=2,707 ; 15,04 \%$ de variance expliquée; alpha =.73). Un dernier facteur comprend 5 items d'incivilités sociales qui sont plus fréquentes entre pairs, comme ceux qui bousculent (item 19) ou ceux qui ne respectent pas les files d'attente (item 15) (valeur propre $=2,506 ; 13,92 \%$ de variance expliquée ; alpha $=.66$ ).

31 Ces 3 catégories d'incivilités - environnementales, scolaires et sociales entre pairs - ont été retenues dans l'analyse des résultats sous forme de moyennes des items impliqués dans la construction de chacune d'elles. 


\begin{tabular}{|c|c|c|c|}
\hline & $\begin{array}{c}\text { FACTEUR } \\
18,09 \% *\end{array}$ & $\begin{array}{c}\text { FACTEUR 2 } \\
15,04 \% *\end{array}$ & $\begin{array}{c}\text { FACTEUR } 3 \\
13,92 \%{ }^{*}\end{array}$ \\
\hline 2. Coller son chewing-gum sous les tables & .537 & & \\
\hline 4. Dégradation du mobilier & .619 & & \\
\hline 6. Tags & .710 & & \\
\hline 10. Brûler le sol en écrasant son mégot & .524 & & \\
\hline 12. Graver les tables & .744 & & \\
\hline 20. Graffittis dans les sanitaires & .714 & & \\
\hline 22. Jeter des papiers par terre & .574 & & \\
\hline 3. Insolence envers les enseignants & & .688 & \\
\hline 7. Insultes graves & & .651 & \\
\hline 9. Manquer les cours sans excuse & & .505 & \\
\hline 13. Perturbations des cours & & .555 & \\
\hline 16. Vente de drogue dans le lycée & & .635 & \\
\hline 18. Comportements provocants & & .471 & \\
\hline 5. Ne pas dire bonjour & & & .458 \\
\hline 14. Élèves qui crachent & & & .552 \\
\hline 15. Élèves qui ne respectent pas les files d'attente & & & .655 \\
\hline 19. Élèves qui bousculent & & & .765 \\
\hline 21. Agressivité verbale entre élèves & & & .573 \\
\hline
\end{tabular}

Analyse en composantes principales de l'échelle d'incivilités (Saturations des items après rotation Varimax)

Principal components analysis of the anti-social behavior scale (Item saturations after Varimax rotation)

\section{Résultats}

\section{Analyse globale des incivilités aux lycées}

Un premier niveau d'analyse a consisté à décrire le niveau des incivilités en termes de fréquence et d'excusabilité à partir d'une moyenne globale des échelles de fréquence et d'excusabilité, et à comparer les réponses des élèves de lycée défavorisé versus défavorisé.

\section{Fréquence des incivilités}

Dans l'ensemble, les incivilités sont perçues comme assez peu fréquentes par les lycéens : la moyenne de 2,59 est comprise entre les réponses «parfois » et « souvent » de l'échelle en 5 points. La comparaison de la perception de la fréquence des incivilités des élèves des établissements favorisés Versus défavorisés par une analyse de variance à un facteur met en évidence l'existence d'une différence significative dans les réponses de ces 2 groupes de sujets: les incivilités sont perçues comme plus fréquentes dans les établissements favorisés que dans les établissements défavorisés [m (lycées favorisés) $=2,69 / \mathrm{m}$ (lycées défavorisés) $=2,49 ; \mathrm{F}=23,113 ; \mathrm{p}<.0001]$.

\section{Excusabilité des incivilités}

Globalement, les incivilités sont jugées comme plutôt inexcusables par les lycéens puisque que la moyenne générale à l'échelle d'excusabilité des incivilités est égale à 2,95. Il convient ensuite de décrire les réponses données par les lycéens quand la consigne leur signifiait de se mettre à la place de leurs professeurs, puis de leurs parents. Des 
comparaisons de moyennes appariées 2 à 2 (consigne pour soi - pour les enseignants / pour soi - pour les parents / pour les enseignants - pour les parents) ont toutes mises en évidence des écarts significatifs (à $p<.0001)$ : les incivilités sont perçues comme les moins excusables pour les enseignants, puis pour les parents, et enfin pour soi (cf. tableau 2).

Tableau 2/Table 2

\begin{tabular}{|c|c|c|c|c|c|c|}
\hline & \multicolumn{2}{|c|}{ POPULATION TOTALE } & \multicolumn{2}{|c|}{$\begin{array}{l}\text { ÉLĖVES DE LYCÉES } \\
\text { DÉFAVORISÉS }\end{array}$} & \multicolumn{2}{|c|}{$\begin{array}{c}\text { ÉLÈVES DE LYCÉES } \\
\text { FAVORISÉS }\end{array}$} \\
\hline & MOYENNE & $\begin{array}{l}\text { ÉCART- } \\
\text { TYPE }\end{array}$ & MOYENNE & $\begin{array}{l}\text { ÉCART- } \\
\text { TYPE }\end{array}$ & MOYENNE & $\begin{array}{l}\text { ÉCART- } \\
\text { TYPE }\end{array}$ \\
\hline $\begin{array}{l}\text { Excusabilité des incivilités } \\
\text { pour soi } \\
\text { pour les enseignants } \\
\text { pour les parents }\end{array}$ & $\begin{array}{l}2,95 \\
3,50 \\
3,32\end{array}$ & $\begin{array}{l}0,44 \\
0,40 \\
0,47\end{array}$ & $\begin{array}{l}3,01 \\
3,54 \\
3,38\end{array}$ & $\begin{array}{l}0,45 \\
0,39 \\
0,47\end{array}$ & $\begin{array}{l}2,88 \\
3,45 \\
3,26\end{array}$ & $\begin{array}{l}0,42 \\
0,39 \\
0,46\end{array}$ \\
\hline
\end{tabular}

Moyennes et écarts-types de l'excusabilité des incivilités dans la population totale et dans les populations de lycées défavorisés et favorisés Means and standard deviations of the excusability of anti-social behavior (Total sample, underprivileged pupils, and privileged pupils)

L'analyse comparative des réponses des élèves de lycée favorisé versus défavorisé en termes d'excusabilité des incivilités met, elle aussi, en évidence des écarts de moyennes significatifs qui vont tous dans le même sens (cf. tableau 2) :

- au niveau de la consigne pour soi : les élèves de lycées défavorisés estiment les incivilités moins excusables que les élèves de lycée favorisé $(t=4,164 ; d d l=749 ; p<.0001)$;

- au niveau des deux autres consignes d'estimation du jugement des adultes (enseignants et parents) : dans ces situations aussi, les élèves de lycées défavorisés estiment que leurs enseignants et leurs parents jugent comme moins excusables les incivilités que ne l'estiment les élèves de lycée favorisé (pour la consigne « enseignants » : $t=3,287 ; d d l=794 ; p=.001$ ) - pour la consigne « parents » $t=3,628 ; d d l=790 ; p<.0001$ ).

\section{Analyse de l'excusabilité des incivilités selon les 3 catégories distinguées : incivilités scolaires, environnementales et sociales}

À ce deuxième niveau d'analyse, afin de tester l'hypothèse d'une différenciation dans l'évaluation des types d'incivilités en fonction du lycée d'appartenance, une analyse de variance à un facteur (lycée favorisé/défavorisé) avec mesures répétées selon le type d'incivilités (environnementales, scolaires et sociales) a permis de dégager les résultats suivants (cf. graphique 1). 


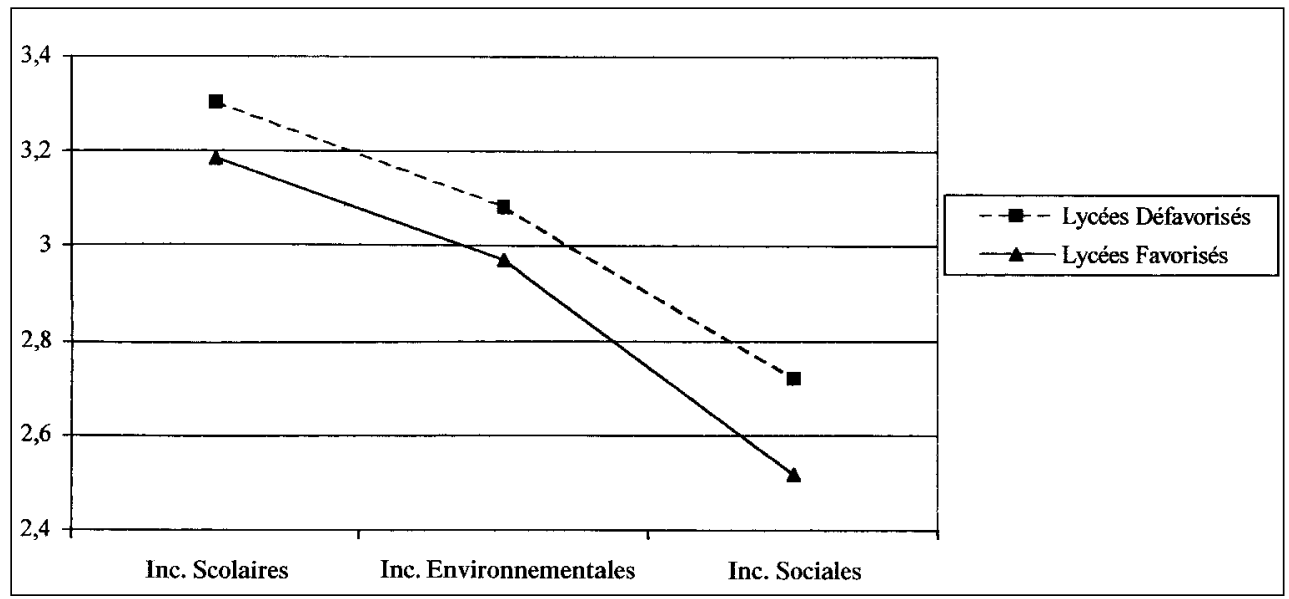

Moyennes d'excusabilité des incivilités scolaires, environnementales et sociales en fonction du type de lycée

Means of the excusability of school, environmental and anti-social behavior according to the type of secondary school

- Les effets inter-sujets mettent en évidence que les élèves des lycées défavorisés jugent l'ensemble des incivilités comme moins excusables que les élèves des lycées favorisés ( $F$ $=18,930 ; d d l=1 / 767 ; p<.0001)$.

- Les effets intra-sujets, qui permettent de comparer les réponses des élèves en fonction du type d'incivilités, sont eux aussi très marqués $(F=502,334 ; d d l=2 / 1534 ; p<.0001)$. Sont considérées comme les moins excusables les incivilités scolaires, puis les incivilités environnementales, et enfin les incivilités sociales.

- Un effet d'interaction entre le type d'incivilités et le type de lycée est aussi à noter ( $F$ $=3,144 ; d d l=2 / 1534 ; p=.043):$ la comparaison des deux lycées est à mettre en relation avec le type d'incivilités. Des tests $\mathrm{T}$ a posteriori révèlent que les écarts entre les 2 types de lycées en termes d'excusabilité sont les plus forts pour les incivilités sociales entre pairs (les élèves de lycées défavorisés jugent beaucoup moins excusables ce type d'incivilités que les élèves de lycées favorisés $-p<.0001$ ) et qu'ils sont les plus faibles pour les incivilités environnementales (l'écart de réponses entre les 2 lycées est toujours significatif, mais il est moins important $-p=.006$ ).

\section{Analyse de la normativité adolescente}

Dans ce troisième niveau d'analyse, pour mettre en évidence enfin une normativité adolescente en matière d'incivilités, normativité spécifique qui se différencierait de celles des adultes, nous avons calculé les écarts entre les moyennes d'excusabilité obtenues : écarts entre lycéens et enseignants, entre lycéens et parents et, enfin, entre enseignants et parents exprimés en termes de calculs en valeur absolue des différences des moyennes d'excusabilité des incivilités pour chaque lycéen.

Rappelons les questions posées initialement : $y$-a-t-il une distance normative à l'égard des adultes affirmée par les adolescents et dans quelles mesures varie-t-elle en fonction de l'établissement fréquenté ? Les adultes sont-ils différenciés selon leur statut, enseignants ou parents? Cette différence varie-t-elle en fonction de l'établissement fréquenté ? 
Une analyse de variance à un facteur (établissement favorisé versus défavorisé) avec mesures répétées réalisées sur les moyennes des écarts en valeur absolue entre adolescents et enseignants, adolescents et parents, et enfin enseignants et parents met en évidence les résultats suivants (cf. graphique 2).

- Les distances en termes d'appréciation de l'excusabilité des incivilités sont différemment marquées par les adolescents $(F=126,678 ; d d l=2 / 1384 ; p<.0001)$ : les écarts entre lycéens et enseignants sont plus importants que les autres (distance moyenne de 0,57 points). L'écart moyen suivant est celui qui existe entre soi et les parents (écart moyen $=0,43$ ), puis vient ensuite l'écart le plus faible, entre adultes, entre enseignants d'une part et parents d'autre part : l'écart est de 0,31 points.

- Un effet tendanciel d'interaction entre ces types d'écarts et l'établissement scolaire d'appartenance apparaît dans l'analyse $(F=2,138 ; d d l=2 / 1384 ; p=.11)$ : les élèves des deux établissements se distinguent dans l'écart marqué entre soi et les enseignants, alors que la distance qu'ils expriment entre eux et leurs parents, et entre enseignants et parents est sensiblement identique. Les tests de comparaison de moyennes a posteriori mettent effectivement en évidence que les élèves de lycées favorisés expriment une distance plus grande à leurs enseignants que ne le font les élèves de lycées défavorisés $(t=2,296 ; p=.02)$.

Graphique 2/Graph 2

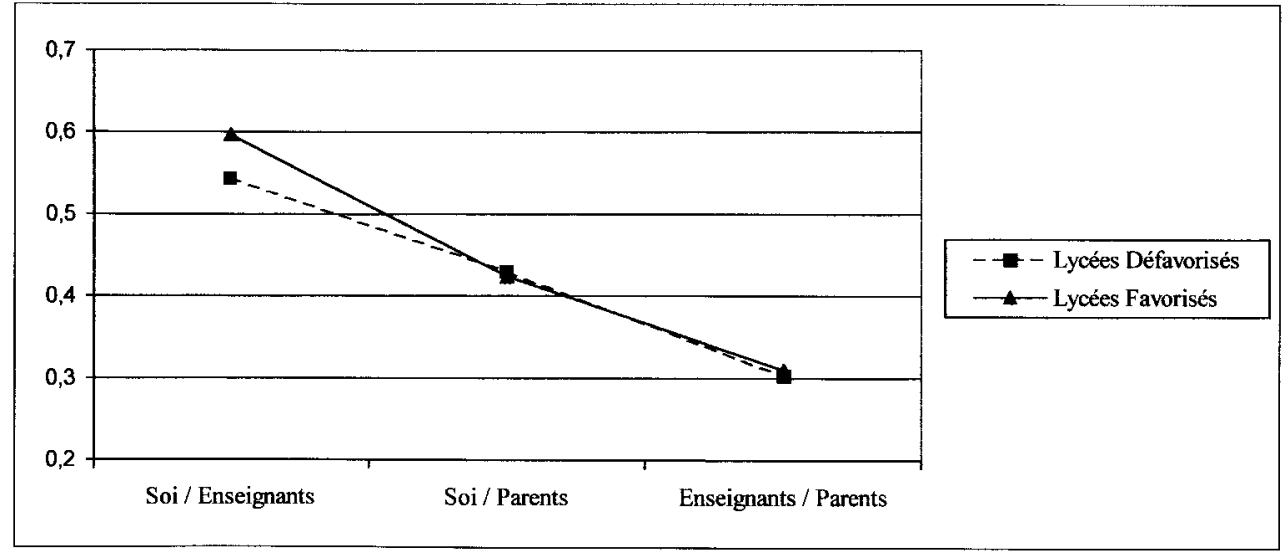

Moyennes des écarts entre les différents acteurs par type d'établissement : écarts soi-enseignants, écarts soi-parents, écarts enseignants-parents

Means of deviation between the different populations according to the different schools : self-teacher deviation, self-parent deviation and teacher-parent deviation

\section{Discussion}

Il ressort des résultats que l'on n'observe aucune banalisation des incivilités de la part des jeunes : en effet, l'ensemble des lycéens jugent les incivilités comme globalement peu excusables. Toutefois, on voit apparaître un effet de l'endogroupe ${ }^{5}$ générationnel, c'est-àdire une normativité adolescente dès lors qu'on envisage l'écart entre les jeunes d'une part, et les enseignants et les parents d'autre part ${ }^{6}$. On a identifié les contours d'une normativité adolescente qui tend à montrer que les adolescents savent que ces comportements contrarient les attentes des adultes, en particulier des enseignants. Dans le lycée, les enseignants incarnent la loi, ils sont toujours perçus comme plus sévères que les parents ${ }^{7}$. 
41 Ainsi, on peut, d'une part, concevoir les incivilités comme des marqueurs identitaires, exprimant une conscience d'appartenance aux groupes de pairs en même temps qu'une revendication existentielle. D'une certaine façon, les adolescents sont confrontés au sentiment d'une double domination : ils se perçoivent comme dominés par les adultes à la fois en tant que jeunes et en tant qu'élèves. En effet, le lycée reste sans doute, à leurs yeux, le seul lieu incarnant encore et si puissamment la coercition, l'effort et la discipline. Dans une telle situation de domination, on comprendra que la revendication d'existence soit forte. On peut, d'autre part, voir les incivilités comme des marqueurs de l'appropriation qui expriment bien un contrôle ou une tentative de contrôle symbolique sur l'espace lycée. Dans l'établissement scolaire, figurant fondamentalement la contrainte (dont la légitimité est constamment ré-interrogée par les élèves) (Dubet, 1991), les adolescents vont choisir et occuper des espaces intersticiels pour manifester leur existence et leur désir d'autonomie. En tant que marqueurs de l'identité et de l'appropriation donc, on peut poser les incivilités comme une forme de résistance à l'ordre scolaire et, plus généralement, à l'ordre adulte.

Parallèlement, on voit apparaitre un effet de l'endogroupe établissement ${ }^{8}$, c'est-à-dire un effet de l'appartenance sociale venant moduler la normativité adolescente puisque l'écart entre adolescents et enseignants varie en fonction de l'établissement fréquenté. De façon comparative, on observe une différence selon l'établissement fréquenté concernant la distance entre les adolescents et les enseignants : c'est dans les établissements les plus favorisés que la distance soi-enseignants est la plus forte, et c'est dans les plus défavorisés qu'elle est la plus faible.

43 Nos résultats mettent au jour une plus grande normativité des élèves de milieu défavorisé - dont on stigmatise le plus souvent l'absence de repères. Quelle que soit la situation, les jeunes les plus défavorisés jugent les incivilités, c'est-à-dire les manquements à la norme, comme moins excusables que les autres. Manifestement, plus on est défavorisé, plus on est assujetti à une normativité stricte, plus on est favorisé, plus on s'en affranchit.

La sous-estimation de la fréquence des incivilités par les lycéens défavorisés pourrait s'expliquer de deux façons :

- D'abord parce que le lycée reste pour eux une source majeure d'identité relativement positive (ce qui n'est pas forcément le cas du collège où les processus de sélection n'ont pas encore été mis en œuvre) et de conscience d'appartenance au groupe de pairs. Cette identification à l'espace du lycée peut conduire effectivement à une sous-estimation des incivilités. On retrouve ce mécanisme dans le cas des étudiants qui s'approprient fortement l'espace de leurs études (Félonneau, 1997).

- Minimiser les incivilités peut renvoyer aussi à une stratégie d'auto-présentation permettant d'éviter le dénigrement et la stigmatisation. Sous-estimer la fréquence des incivilités tout en affirmant leur non-excusabilité, permet probablement de sauvegarder une identité sociale positive. En effet, ces jeunes vivent dans des secteurs urbains défavorisés dont l'image est relativement dévalorisée. On imagine alors qu'ils affichent une conformité d'autant plus évidente qu'ils sont soucieux d'éviter une catégorisation défavorable autrement dit un effet de « mauvaise réputation» (Croizet \& Leyens, 2003) ! En outre, les membres des catégories dominées apparaissent toujours plus soumis aux impératifs normatifs (Bourdieu, 1979 ; Lorenzi-Cioldi, 2002) et désireux de ne pas s'écarter de la norme dominante.

En revanche, les adolescents de milieu favorisé ont une propension supérieure à s'affranchir de la norme des adultes enseignants. Inscrits dans les lycées les plus favorisés, et occupant, de fait, une position socialement dominante, ils n'ont, pour ainsi 
dire, rien à prouver en termes de conformisation. Alors qu'il est fréquent d'évoquer l'indiscipline et l'insoumission des élèves issus de milieu populaire, c'est paradoxalement dans les lycées socialement favorisés, situés dans des espaces urbains socialement valorisés, que l'on observe dans nos résultats une norme explicite de prise de distance à l'égard des enseignants. On reconnaît ici la plus grande marge de manœuvre des individus en position dominante qui peuvent - et doivent savoir - s'affranchir dans certains cas de la norme ordinaire pour se poser en individu singulier. Cet apprentissage de l'autonomie et de l'originalité s'observe davantage dans les milieux favorisés où la norme d'éducation est plus libérale, le moratoire adolescent - au sens d'une période accordée par la société pour l'expérimentation de nouveaux rôles sociaux (Erikson, 1972) - est plus admis.

Retenons qu'en termes de gravité, les types d'incivilités sont évalués différemment : les moins excusables sont les incivilités scolaires, puis viennent les incivilités environnementales et enfin les incivilités sociales entre pairs.

Moins excusables que les autres, les incivilités scolaires renvoient à des comportements visibles susceptibles d'être immédiatement sanctionnés par les professeurs. On retrouve là le rapport aux enseignants qui incarnent la loi.

À l'inverse des autres incivilités qui constituent des incivilités d'interaction, les incivilités environnementales sont plus irréversibles. En effet, elles laissent une trace négative dans l'espace, une forme de "souillure » du territoire commun qui s'avère plus intolérable qu'une agression envers un pair. La psychologie sociale de l'environnement insiste largement sur l'appropriation symbolique du territoire. Or, si le lycée incarne bien la spatialisation de l'identité scolaire, il est investi émotionnellement de façon très puissante. De ce fait, la dégradation que l'espace ainsi approprié peut subir est vécue comme une véritable "offense territoriale ", comme une forme de "contamination" (Goffman, 1973). À partir du moment où le sujet se projette dans un espace donné, celui-ci ne saurait être "profané». Chaque trace d'incivilité pourrait être vécue comme menaçante pour l'identité (Ségaud, 1992).

Globalement, enfin, il ressort de cette recherche que les jeunes, dans leur ensemble, identifient parfaitement les usages en vigueur et les normes « dominantes", ce qui ne les empêche aucunement de les transgresser dans une quête d'affirmation de soi et de reconnaissance par les pairs. Au contraire, les incivilités peuvent apparaittre comme des tentatives de subversion de l'ordre scolaire, et de l'ordre adulte en général, relativement normées et codifiées. La mise en évidence d'une normativité adolescente en matière d'usage de l'espace permet de dépasser l'idée d'une anomie généralisée et d'une perte de repères grandissante en montrant que le lycée demeure encore largement un espace qui fait sens. 


\section{BIBLIOGRAPHIE}

Altman, I. (1975). The environment and social behavior privacy personal space, territories, crowding. Monterey : Brooks, Cole.

Augustin, J.-P. (1994). Surf Atlantique. Les territoires de l'éphémère. Talence : Maison des Sciences de l'Homme d'Aquitaine.

Bernard, Y. (1997). La représentation de la civilité dans la société urbaine d'aujourd'hui. Psychologie Française, 42, 2, 113-122.

Bourdieu, P. (1979). La distinction. Paris : les Éditions de Minuit.

Brown, B. B. (1990). Peer groups and peer cultures. In S. S. Feldman \& G. R. Elliott (Eds.), At the threshold: the developing adolescent (pp. 171-196). Cambridge MA, UK : Harvard University Press.

Buhrmester, D. (1996). Need fulfillment, interpersonal competence, and the developmental contexts of early adolescent friendship. In W. Bukowski, A. Newcomb, \& W. Hartup (Eds.), The company they keep (pp. 158-185). New-York, U.S.A. : Cambridge University Press.

Chazel, F. (2004). Article « Normes Sociales ». Encyclopédia Universalis.

Cloutier, R. (1996). Psychologie de l'adolescence (2 édition). Montréal, Québec, Canada : Gaëtan Morin.

Conger, J. J. (1991). Adolescence and youth (4e édition). New-York, U.S.A. : Harper Collins

Coslin, P. G. (1999). Les adolescents devant les déviances. Paris : P.U.F.

Coslin, P. G. (2002). Psychologie de l'adolescent. Paris : Armand Colin.

Croizet, J.-C., \& Leyens, J.-P. (2003) Mauvaises réputations. Réalités et enjeux de la stigmatisation sociale . Paris : Armand Colin.

Debarbieux, E. (1996). La violence en milieu scolaire. Paris : P.U.F.

Dubet, F. (1991). Les lycéens. Paris : Le Seuil.

Dumora, B. (1998). Expérience scolaire et orientation. L'Orientation Scolaire et Professionnelle, 27, 2, 211-234.

Ehrenberg, A. (1998). La fatigue d'être soi. Paris : Odile Jacob.

Erikson, E.-H. (1972). Adolescence et crise : la quête de l'identité. Saint-Armand : Flammarion.

Farrington, D. (1995). The development of offending and antisocial behaviour from childhood : key findings from the Cambridge Study in Delinquent Youth. Journal of Child Psychology and Psychiatry, 36, 1-35.

Félonneau, M.-L. (1994) Les étudiants et leurs territoires. Revue Française de Sociologie, 25, 533-559.

Félonneau, M.-L. (1997). L'étudiant dans la ville. Paris : L'Harmattan.

Félonneau, M.-L., \& Busquets, S. (2001). Tags et grafs... Les jeunes à la conquête de la ville. Paris :

L'Harmattan.

Fischer, G.-N. (1989). Psychologie des espaces de travail. Paris : Armand Colin. 
Fischer, G.-N. (1992). Psychologie sociale de l'environnement. Toulouse : Privat.

Furman, W., \& Whener, E. A. (1997). Adolescent romantic relationships : a developmental perspective. In S. Shulman \& W. A. Collins (Eds.), Romantic relationships in adolescence:

developmental perspectives. New Direction for Child Development, $\mathrm{n}^{\circ}$ 78. San Francisco, U.S.A. : Jossey-Bass.

Furstenberg, F. (1971). Public reaction to crime in the streets. American Scholar, 40, 601-610.

Goffman, E. (1973). La mise en scène de la vie quotidienne. Paris : Éditions de Minuit.

Kohlberg, L. (1969). Stage and sequence : the cognitive-developmental approach to socialization. In D. A. Goslin (Ed.), Handbook of socialization theory and research (pp. 347-480). Chicago, U.S.A. : Rand McNally College Publishing Company.

Lagrange, H. (1984). La perception de la violence dans l'opinion publique. Revue Française de Sociologie, 25, 636-657.

Lagrange, H. (1995). La civilité à l'épreuve. Crime et sentiment d'insécurité. Paris : P.U.F.

Lannegrand-Willems, L. (2000). Identité scolaire et effets de contexte chez l'adolescent. Thèse de Doctorat de l'Université Victor Segalen Bordeaux 2.

Leblanc, M., \& Frechette, M. (1983). Une théorie intégrative de la régulation de la conduite délinquante. Annales de Vaucresson, 20, I, 34.

Lipiansky, E.-M. (1993). La problématique identitaire à l'adolescence. Revue de Psychologie de la Motivation, 16, 43-54.

Lorenzi-Cioldi, F. (2002). Les représentations des groupes dominants et dominés. Grenoble : P.U.G.

Maffesoli, M. (2000). Le temps des tribus. Le déclin de l'individualisme dans les sociétés post-modernes. Paris : La Table Ronde.

Moser, G., \& Lidvan, P. (1991). Environnement urbain et sentiment d'insécurité. In Y. Bernard \& M. Segaud (Éds.), La ville inquiète, habitat et sentiment d'insécurité, La Garenne-Colombe : Les Éditions de l'Espace Européen.

Py, J., \& Somat, A. (1991). Normativité, conformité et clairvoyance : leurs effets sur le jugement évaluatif en contexte scolaire. In J.-L. Beauvois, R.-V. Joule, \& J.-M. Monteil (Éds.), Perspectives cognitives et conduites sociales $3:$ Quelles cognitions? Quelles conduites ? Cousset, Suisse : DelVal.

Riffault, H. (Éd.) (1993). Les systèmes de valeurs des Français. Paris : P.U.F.

Roché, S. (1996). La société incivile. Paris : Le Seuil.

Roché, S. (1998). Sociologie politique de l'insécurité. Paris : P.U.F.

Rouleau-Berger, L. (1991) La Ville-Intervalle, jeunes entre centre et banlieue. Paris : Méridiens Kliensieck.

Scardigli, V. (1987). L’Europe des modes de vie. Paris : Éditions du C.N.R.S.

Ségaud, M. (Éd.) (1992). Le propre de la ville : pratiques et symboles. La Garenne-Colombe : Les Éditions de l'Espace Européen.

Sélosse, J. (1997) Adolescences, violence et déviances. Vigneux : Éditions Matrice.

Sherif, M. (1965). Influence du groupe sur la formation des normes et des attitudes. In A. Lévy (Éd.), Psychologie Sociale. Textes fondamentaux anglais et américains. Paris : Dunod.

Steinberg, L. (1999). Adolescence (5e édition). Boston, U.S.A. : McGraw-Hill. 
Steinberg, L., \& Morris, A. (2001). Adolescent development. Annual Review of Psychology, 52, 83-110.

Stoetzel, J. (1983). Les Valeurs du Temps Présent. Paris : P.U.F.

Wievorka, M. (1999). Violence en France. Paris : Le Seuil.

\section{NOTES}

1. Cet article présente une recherche qui s'inscrit dans une réflexion plus vaste portant sur les adolescents en milieu urbain. Elle est menée dans le cadre du Laboratoire de Psychologie de l'Université Victor Ségalen Bordeaux 2 et du programme quadriennal de recherche de la Maison des Sciences de l'Homme d'Aquitaine (1998-2002).

2. Dans le langage sociologique, une norme constitue une règle ou un critère régissant notre conduite en société. Il ne s'agit pas d'une régularité statistique dans les comportements observés, mais d'un modèle culturel de conduite auquel nous sommes censés nous conformer. La norme acquiert une signification sociale dans la mesure où, comme le fait ressortir le terme de culturel, elle est - jusqu'à un certain point - partagée (Chazel, 2004).

3. Au sens où l'entend Altman de territoires où s'expriment des relations secondaires (de type professionnel ou amical par exemple), appropriés de façon durable par des groupes définis, par opposition aux territoires primaires (associés aux relations familiales) et aux territoires publics.

4. Dans ces recherches, la notion d'excusabilité permet de repérer les limites et l'évolution de la tolérance et de la permissivité quant à un certain nombre d'actes déviants, plus ou moins répréhensibles.

5. Ici l'endogroupe pour les lycéens est celui des jeunes de leur âge, l'exogroupe est celui des adultes.

6. On peut penser que l'ordre de passation des consignes extrêmisent les réponses. Néanmoins, cet ordre reste pertinent du fait que les adolescents répondent pour eux-mêmes en premier, c'est-à-dire sans comparaison à l'adulte et qu'on obtient dans les résultats des moyennes brutes relativement élevées. Or, si nous avions contrebalancé les ordres de questionnement, les adolescents amenés à répondre par rapport à soi après avoir répondu par rapport aux parents et/ou aux enseignants auraient peut-être été amenés à différencier davantage leurs propres jugements de ceux des adultes.

7. Ce qui s'explique notamment par le fait que l'enquête est passée dans le lycée, et donc que l'autorité enseignante est rendue plus saillante.

8. L'endogroupe établissement renvoie à l'ensemble des jeunes interrogés dans le même lycée.

\section{RÉSUMÉS}

À partir d'un prisme associant la psychologie sociale environnementale et la psychologie du développement, nous proposons une lecture psychosociale de l'inscription de l'adolescent dans le lycée comme espace majeur de sa vie quotidienne. Grâce à l'étude des représentations des incivilités rencontrées dans le lycée (étude sur 842 élèves fréquentant des lycées sociologiquement contrastés), on a mis en évidence une normativité adolescente différente de celle des adultes, pour autant, on n'observe aucune banalisation des comportements incivils. De 
plus, pour les jeunes, une incivilité environnementale, c'est-à-dire une trace négative dans l'espace, est moins excusable qu'une incivilité envers autrui. Nos résultats ont également mis au jour une plus grande normativité des élèves de milieu défavorisé : les jeunes les plus défavorisés jugent les incivilités, c'est-à-dire les manquements à la norme, comme moins excusables que les autres. Globalement, enfin, il ressort de cette recherche que les adolescents identifient parfaitement les usages en vigueur et les normes « dominantes », tout en s'en distanciant.

Based on a prism combining Social Environmental Psychology and Developmental Psychology, we propose a psychosocial view of the adolescent's place in secondary school as a major area of his everyday life. From a study of the representations of anti-social school behavior (using a sample of 842 pupils from socially contrasted secondary schools), we showed that an adolescent normativity exists, different to that of adults, but the antisocial behavior was not trivialized. In addition, for the pupils environmental anti-social behavior was less excusable than anti-social behavior towards another person. Our results also pointed out a more pronounced underprivileged pupil normatively: they assess anti-social behavior-that is the breaches of the norm-as less excusable than the other pupils. Overall, we showed that adolescents accurately identified the behavior in use and the "dominant" norms, whilst at the same time distancing themselves from them.

\section{INDEX}

Mots-clés : Adolescence, Identité, Incivilités, Normativités scolaires

Keywords : Anti-social behavior, Identity, School normativities

\section{AUTEURS}

\section{MARIE-LINE FÉLONNEAU}

Maître de conférences H.D.R. en psychologie sociale à l'Université Victor Segalen Bordeaux 2. Ses travaux portent sur la thématique « Cognition sociale et Environnement » et plus particulièrement sur les représentations sociales de l'urbain. Membre du Laboratoire de Psychologie EA 3662 (Université Victor Segalen Bordeaux 2, 3 ter, place de la Victoire, 33076 Bordeaux Cedex).

\section{LYDA LANNEGRAND-WILLEMS}

Maître de conférences en psychologie du développement à l'Université Victor Segalen Bordeaux 2. Ses travaux portent sur les aspects psychosociaux du développement à l'adolescence. Membre du Laboratoire de Psychologie EA 3662. 